\title{
Sex differences in the working memory of students in Ahmadu Bello University, Zaria, Nigeria using the N-back task
}

\author{
${ }^{1}$ Tende, J. A., ${ }^{2}$ Eze, E. D. ${ }^{3}$ Yusuf, A., ${ }^{4}$ Malgwi, I. S. and ${ }^{5}$ Wilcox, B. \\ Departmen t of Human Physiology, Faculty of Medicine, Ahmadu Bello University, Zaria, Nigeria.
}

\begin{abstract}
The study of sex differences in cognition has been shrouded with much controversy. Working memory is a central construct in cognitive neuroscience. This study examined sex differences in working memory of students in Ahmadu Bello University, Zaria using the N-back task. To achieve this aim, fifty four (54) students of Ahmadu Bello University in the age range of 18-35 years consisting of 31 females and 23 males were used for the study. The subjects were screened for some confounding variables such as blood pressure profiles, body mass index that affect cognition. Cognitive functions were assessed with the Mini Mental State Examination Test, while working memory was tested with the N-back task. Results obtained from the N-back scores showed that there was no statistically significant difference $(p>0.05)$ in working memory sexes, with mean values of $75.09 \pm 0.63$ for males and $73.81 \pm 0.58$ for females. The mini-mental state examination also revealed no statistically significant difference $(p>0.05)$ between the both sexes with a mean values of $29.52 \pm 0.12$ for males and $29.07 \pm 0.20$ for females. The mean BMI of the male and female population was $22.24 \pm 0.47 \mathrm{~kg} / \mathrm{m}^{2}$ and $22.36 \pm 0.88 \mathrm{~kg} / \mathrm{m}^{2}$ respectively and was within the range accepted as normal. The present work revealed no sex differences in working memory using n-back task between males and females in the studied population.
\end{abstract}

Keywords: Cognition, Blood pressure, Mini-Mental State, N-back score, Sex difference, working memory

\section{Introduction}

Cognition refers to the mental activities involved in the acquisition, processing, organization and the use of knowledge. These mental activities include the following components of cognitive function; selective attention, perception, working memory, logical reasoning and problem-solving [1]. Working memory may be defined as the system for the temporary maintenance and manipulation of information necessary for the performance of such complex cognitive activities such as comprehension, learning and reasoning. It is a major component of cognition [2]. The study of working memory has become a focal point of memory research in the past 2 decades [3]. Working memory affects all areas of living from the kindergarten to the college [4]. The Nback task is a working memory task. It involves a set of information to be remembered along with a secondary processing task [5]. Working memory plays an essential role in complex cognition. Everyday, cognitive tasks such as reading a newspaper article, calculating the appropriate amount to tip in a restaurant, mentally rearranging furniture in ones living room to create space for a new sofa and comparing and contrasting various attributes of different apartments to decide which to rent often involve multiple steps with intermediate task results that need to be kept in mind temporarily to accomplish the task at hand successfully [6]. Working memory is a central construct in cognitive psychology and cognitive neuroscience [6]. Working memory is the system responsible for the temporary maintenance and manipulation of information, necessary for the performance of such complex cognitive functions as learning, comprehension and reasoning [2]. The aim of the present work was to evaluate the sex differences in the working memory of students in Ahmadu Bello University, Zaria, Nigeria, using the N-back task.

\subsection{Materials used}

\section{Materials And Methods}

Sphygmomanometer and stethoscope were used to assess blood pressure. Clinical thermometer was used to measure body temperature. Cotton wool and alcohol were used to sterilize the thermometer. Pulse rate was measured using a stop watch. Measuring tape was used to measure height; weighing scale was used to measure weight. BMI was computed from values of weight and height. Computerized software program and computer laptop was used to administer the N-back task.

\subsection{Study location and population}

The work was carried out within the campuses of Ahmadu Bello University, Zaria, Nigeria in the Month of December, 2009. The population for this study was a finite population that comprised 54 subjects: (33 females, 21 males) students of Ahmadu Bello University Zaria within the age group of 18-35 years old. The subjects selected for the study were screened for confounding variables. 


\subsection{Screening of subjects}

The baseline for the screening of subjects was obtained using a questionnaire and physical examination. The inclusion criteria for the subjects were normal blood pressure (systolic blood pressure $\leq$ $140 \mathrm{mmHg}$, diastolic blood pressure $\leq 90 \mathrm{mmHg}$ ) good health status, normal body temperature $936.50 \pm 0.05^{\circ} \mathrm{C}$ ), Body mass index $\left(20 \geq\right.$ BMI $\left.\leq 25 \mathrm{~kg} / \mathrm{m}^{2}\right)$. Exclusion criteria included: hypertension (blood pressure $\geq 140 / 90 \mathrm{mmHg}$ ), hypertension blood pressure $\leq 90 / 60 \mathrm{mmHg}$ ), overweight (BMI $\geq 25 \mathrm{~kg} / \mathrm{m}^{2}$ ), underweight (BMI $\leq 20 \mathrm{k} / \mathrm{gm}^{2}$ ), hyperthermia (body temperature $\geq 38^{\circ} \mathrm{C}$ ), hypothermia (body temperature $\leq 35.5^{\circ} \mathrm{C}$ ), history of mental health disease in family, use of alcohol, use of tobacco, use of psychoactive substances, use of any medication and sickness.

\subsection{Data collection techniques used}

A random sampling was done among the volunteer students that met the baseline criteria (as determined by the questionnaire and physical examination) were used as subjects for the study. Fifty four (54) students of Ahmadu Bello University in the age range of 18-35 years consisting of 31 females and 23 males formed the sample size of the study. A questionnaire was used to assess suitability of the volunteers for the research. A bio-data form and pen were used to fill in the results of the physical examination.

\subsection{Experimental protocol}

The volunteers were made to complete the questionnaire. Physical and physiologic parameters were assessed. The neuropsychological tests-the mini-mental state examination and the N-back task were carried out.

\subsection{Neuropsychological tests}

\subsubsection{Mini-Mental State Examination (MMSE)}

The MMSE is considered to provide a global assessment of cognitive functions. It is one of the most widely used clinical instruments for, quickly detecting cognitive impairment and assessing its severity as well as for monitoring cognitive changes over time. The MMSE consists of a variety of questions that can be administered in five to ten minutes. The questions are designed to elicit information about orientation, registration, attention, calculation, ability, recall language and praxis. Scoring of MMSE consists of a total number of correct answers. Refusal to answer implies inability to answer correctly. The maximum is 30 . A scope of 24-30 is generally classified as no cognitive impairment, 18-23 as mild cognitive impairment and 0-17 as a severe cognitive impairment [7].

\subsubsection{N-back Task}

$\mathrm{N}$-back was the neuropsychological test used to assess working memory was that adopted and modified by Ernst $e t$ al., (2001) [8]. This is a computerized test. The participants were required to keep in memory, a series of letters that are constantly updated. The letters appear in the middle of the computer screen, one at a time every 2 seconds. Eighty (80) letters were programmed as such. The volunteers were instructed to say 'target' whenever there was the repetition of a letter with exactly one intervening letter and remain silent when any other letter appeared.

\subsection{Ethical considerations}

The experimental protocols for the study were explained to the volunteers. Consent was obtained from the volunteers. The volunteers were assured that the information provided was confidential and their identity anonymous.

\subsection{Statistical analysis}

Data obtained from baseline assessment and characteristics, N-back tasks and performance scores in mini-mental state examination test were presented as mean \pm SEM. Gender differences from empirical values for the neuropsychological tests were compared between the two groups using independent- $t-$ test. The difference among the means were considered statistically significance at $\mathrm{p}<0.05$.

\section{Results}

"Table" 1 showed that there was no statistically significant difference $(\mathrm{p}>0.05)$ in all base line assessment and characteristics between the males and females when compared. There was also no significant difference ( $>0.05)$ in all blood pressure components between the males and females when compared ("Table" 2). The results obtained showed that there was no statistically significant difference $(p>0.05)$ in the cognitive function 
and working memory assessed between the males and females using the performance score in mini-mental state examination test and $\mathrm{N}$-back task score when compared as shown in "Table" 3.

Table 1: Mean \pm SEM of base line assessment and characteristics of subjects

\begin{tabular}{|l|l|l|}
\hline & Males & Females \\
\hline Age (Years) & $25.04 \pm 0.50^{\mathrm{ns}}$ & $23.45 \pm 0.37^{\mathrm{ns}}$ \\
\hline Body temperature $\left({ }^{\circ} \mathrm{C}\right)$ & $36.43 \pm 0.11^{\mathrm{ns}^{-}}$ & $36.42 \pm 0.16^{\mathrm{ns}}$ \\
\hline Height $(\mathrm{m})$ & $1.71=0.02^{\mathrm{ns}^{-}}$ & $1.62 \pm 0.02^{\mathrm{ns}}$ \\
\hline Weight $(\mathrm{Kg})$ & $64.56 \pm 1.33^{\mathrm{ns}}$ & $58.34 \pm 2.44^{\mathrm{ns}}$ \\
\hline Body mass index $\left(\mathrm{Kg} \mathrm{m}^{2}\right)$ & $22.24 \pm 0.47^{\mathrm{ns}^{2}}$ & $22.36 \pm 0.89^{\mathrm{ns}}$ \\
\hline
\end{tabular}

${ }_{2}^{2} \mathrm{p}<0.05$ is statistically significant while ns= not significant when compared

Table 2: Mean \pm SEM of blood pressure components of subjects

\begin{tabular}{|c|c|c|}
\hline & Males & Females \\
\hline Systolic blood pressure (mmHg) & $113.74 \pm 2.20^{\mathrm{ns}}$ & $108.87 \pm 1.76^{\text {ns }}$ \\
\hline Diastolic blood pressure (mmHg) & $74.69=1.88^{\mathrm{ns}}$ & $70.19=1.76 \mathrm{~ns}$ \\
\hline Pulse pressure (mmHg) & $39.04 \pm 1.94^{\mathrm{ns}}$ & $38.68=1.41^{\mathrm{ns}}$ \\
\hline $\begin{array}{l}\text { Mean arterial blood pressure } \\
(\mathrm{mmHg})\end{array}$ & $112.61=2.29 \mathrm{~s}$ & $106.49=2.20 \mathrm{~ns}$ \\
\hline Pulse rate (Beats $/ \mathrm{min})$ & $70.17=1.25^{\mathrm{ns}}$ & $77.09=1.58 \mathrm{~ns}$ \\
\hline
\end{tabular}

? $2<0.05$ is statistically significant while $n s=$ not significant when compared

Table 3: Mean \pm SEM of performance scores in mini-mental state examination and N-back task of subjects

\begin{tabular}{|l|l|l|}
\hline & Males & Females \\
\hline $\begin{array}{l}\text { Mini-mental state examination } \\
\text { test score }\end{array}$ & $29.52 \pm 0.12^{\mathrm{ns}}$ & $29.07 \pm 0.19^{\mathrm{ns}}$ \\
\hline N-back score & $75.08=0.63^{\mathrm{ns}}$ & $73.81=0.58^{\mathrm{ns}}$ \\
\hline \multicolumn{2}{|l}{${ }^{2} \mathrm{p}<0.05$ is statistically significant while ns= not significant when compared }
\end{tabular}

\section{Discussion}

Research on sex differences in cognitive abilities investigates the distribution of cognitive skills between males and females. Such research employs a number of experimental tests of cognition, which take a variety of forms [9]. Cognition refers to the mental activities involved in the acquisition, processing, organization and use of knowledge. These mental civilities include the following components of cognitive function: selective attention, perception, working memory, logical reasoning and problem-solving. Hence cognition is a global term that describes all the mental activities that are engaged in our thoughts [1]. Working memory is a central construct in cognitive psychology and cognitive neuroscience [6]. There are good numbers of working memory tests. However, the test used in this study was the N-back task. This test has been referred to as the gold standard of working memory measures in the field of cognitive neuroscience [3].

\subsection{Baseline Assessment and Characteristics of Subjects}

According to Piaget's theory of cognitive development, individuals within the age bracket of 18-35 belong to the formal operational period and are capable of constructive logical reasoning and scientific deductions [10]. This informed the choice of the selected age group. The mean of the male population (25.04 \pm $0.50)$ years and the female population $(23.45 \pm 0.37$ years) fell within the age range chosen for the study Certain factors such as hypertension and obesity have been negatively correlated with cognition [11]. An individual is classified as hypertensive when the blood pressure is established over time as $\geq 140 / 90 \mathrm{mmHg}$ and classified as obese when BMI is $\geq 25 \mathrm{~kg} / \mathrm{m}^{2}$. The mean values for the males and females for blood pressure and BMI are $114 / 75 \mathrm{mmHg}, 109 / 70 \mathrm{mmHg}, 22.24 \pm 0.47 \mathrm{~kg} / \mathrm{m}^{2}, 22.36 \pm 0.88 \mathrm{~kg} / \mathrm{m}^{2}$ respectively. These values are within the normal range. The subjects had no cognitive impairment as shown by the mean mini-mental score examination test for males $(29.52 \pm 0.12)$ and females $(29.07 \pm 0.19)$. These values are classified for no cognitive impairment [7]. 


\subsection{Sex Differences in Working Memory}

The result of the present study showed that there is no significant sex difference in working memory. It has been established that there are sex differences in cognitive abilities [12]. However, the nature, onset, prevalence and magnitude of such differences vary. There are cognitive functions in which no sex differences are observed [13]. Working memory has components which have subcomponents. Previous work has shown that there are significant differences in spatial tasks and verbal tasks [14]. Sex differences in modal-specific elements of working memory were investigated by utilizing words and pictures as stimuli. The findings on verbal working memory were consistent with previous research and pointed to sex differences in cognitive ability putatively resulting from functional neuro-anatomical dissimilarities [15]. However, on the visual working memory task, women showed significantly greater recall than men, which was inconsistent with previous research and underscored the need for further research.

\section{Conclusion}

The result of this present study suggests that there are no sex differences in cognitive function and working memory between the males and females in the studied population.

\section{References}

[1] M. Yvilsaker, Tutorials in cognitive intervention/rehabilitation, Journal of experimental psychology, Applied, 9, 2006, 23-32.

[2] A.Baddeley, Working Memory: the Interface between Memory and Cognition, Journal of cognitive neuroscience, 4(3), 1992, 281288 .

[3] J. T. Shelton, R. L. Meizger and E. M. Elliot, A group-administered lag task as a measure of working memory, Behaviour Research Methods, 39 (3), 2007, 482-493.

[4] B. J. Alloway,The cognitive and behavioural characteristics of children with low working memory. Child Development, Vol. 80, 2009, 606-621

[5] M. Daneman and P. A. Carpenter, Individual differences in working memory and reading, Journal of Verbal Learning and Verbal Behaviour 19(4), 1980, 450-466

[6] A. Miyake, and P. Shah, Toward a unified theory of working memory: Emerging general consensus, unresolved theoretical issues and future research directions. In Miyake, A. and Shah, P. (eds). Models of working memory: Mechanisms of active maintenance and executive control. Cambridge, Cambridge University Press, 1999, Pp.442-481.

[7] M. F. Folstein, S. E. Folstein, and P. R. McHugh, "Mini-mental state" A practical method for grading the cognitive state of patients for the clinician, J. Psychiatr. Res. 12(3), 1975,189-98.

[8] M. Ernst, J.A. Matochik, S. J. Heishman, J. D. Van Horn, P. H. Jons, J. E. Henningfield and E. D. Jonson, Effect of nicotine on brain activation during performance of a working memory task, Neurobiology, 98(8), 2001, 4728-4733.

[9] Hedges, L.V. and A. Nowell, "Sex Differences in Mental Test Scores, Variability and numbers of High-Scoring Individuals" Science, 269, 1995, 41-45.

[10] G. Claxton, Understanding piaget, Cognitive psychology, Routelege and Kegan Paul 3 (London, Boston, 2002), 26-28

[11] J. M. Starr, I. J. Dreary, H. Fox and L. J. Whalley, Blood pressure and cognition. Gerontology, 53(6), 2007, 432-437

[12] R. Lynn, "Sex differences in intelligence and brain size: a developmental theory" Intelligence, 7, 1999, 1-12.

[13] D. Halpern, Sex brains and hands-gender differences in cognitive abilities, Skeptic, 2(3), 1993, 96-103.

[14] S. B. Kaufman, (2007). Sex differences in mental rotation and spatial visualization ability: can they be accounted for by differences in working memory capacity? Intelligence, 35(30), 2007, 211-223

[15] A. Harness, L. Jacot, S. Scherf, A. White, and J. E Warrick, sex differences in working memory, Psychol Rep.103(1), 2008, 214218 